\title{
The integral Novikov conjectures for linear groups containing torsion elements
}

\author{
Lizhen Ji
}

\begin{abstract}
In this paper, we show that for any global field $k$, the generalized integral Novikov conjecture in both $K$ - and $L$-theories holds for every finitely generated subgroup $\Gamma$ of $\operatorname{GL}(n, k)$. This implies that the conjecture holds for every finitely generated subgroup of $\operatorname{GL}(n, \overline{\mathbb{Q}})$, where $\overline{\mathbb{Q}}$ is the algebraic closure of $\mathbb{Q}$. We also show that for every linear algebraic group $\mathbf{G}$ defined over $k$, every $S$-arithmetic subgroup satisfies this generalized integral Novikov conjecture. We note that the integral Novikov conjecture implies the stable Borel conjecture, in particular, the stable Borel conjecture holds for all the above torsion-free groups. Most of these subgroups are not discrete subgroups of Lie groups with finitely many connected components, and some of them are not finitely generated. When the field $k$ is a function field such as $\mathbb{F}_{p}(t)$, and the $k$-rank of $\mathbf{G}$ is positive, many of these $S$-arithmetic subgroups such as $S L\left(n, \mathbb{F}_{p}[t]\right)$ do not admit cofinite universal spaces for proper actions.
\end{abstract}

\section{Introduction}

The original Novikov conjecture concerns the homotopy invariance of higher signatures of oriented manifolds $M$. If $\Gamma=\pi_{1}(M)$ is the fundamental group of $M$, then the Novikov conjecture is equivalent to the rational injectivity of the assembly map

$$
A^{\langle-\infty\rangle}: H_{*}\left(B \Gamma, \mathbb{L}^{\langle-\infty\rangle}(\mathbb{Z})\right) \rightarrow L_{*}^{\langle-\infty\rangle}(\mathbb{Z}[\Gamma]),
$$

that is, $A^{\langle-\infty\rangle} \otimes \mathbb{Q}$ is injective. (Note that the surgery groups $L^{\langle k\rangle}(\mathbb{Z} \Gamma)$ depend on a decoration $k, 2 \geqslant k \geqslant-\infty$. For each decoration $k$, there is a corresponding assembly map $A^{\langle k\rangle}$. See [43, $44]$ for details.) In the following, we often denote the assembly map $A^{\langle-\infty\rangle}$ by $A$ for simplicity. The injectivity of $A^{\langle-\infty\rangle}$ is called the integral Novikov conjecture for $\Gamma$. This integral Novikov conjecture is useful for various purposes. For example, it allows us to understand part of the difficult and important groups $L_{*}^{\langle-\infty\rangle}(\mathbb{Z}[\Gamma])$ in terms of the generalized homology theory $H_{*}\left(B \Gamma, \mathbb{L}^{\langle-\infty\rangle}(\mathbb{Z})\right.$ ) (see [38] for a comprehensive survey on the Novikov and related conjectures and their applications); it also implies the stable Borel conjecture (see Proposition 2.8 for the precise statement and proof.)

Note that in (1.1), the Novikov conjecture only depends on the group $\Gamma$. If $\Gamma$ contains torsion elements, then $A$ is expected to fail to be injective in general (see $[\mathbf{3 5}, \mathbf{3 8}, \mathbf{4 5}, \mathrm{p} .602])$. In this case, there is an assembly map associated with the universal space $E_{\mathcal{F}} \Gamma$ (which is also often denoted by $\underline{E} \Gamma$ ) for proper actions of $\Gamma$, that is, the universal space associated with the family $\mathcal{F}$ of all finite subgroups of $\Gamma$ :

$$
A: H_{*}^{\Gamma}\left(E_{\mathcal{F}} \Gamma, \mathbb{L}^{\langle-\infty\rangle}(\mathbb{Z})\right) \rightarrow L_{*}^{\langle-\infty\rangle}(\mathbb{Z}[\Gamma]) .
$$

If this assembly map $A$ is injective in (1.2), we say that the generalized integral Novikov conjecture in $L$-theory holds for $\Gamma$.

Similarly, there is an assembly map in algebraic $K$-theory:

$$
A: H_{*}^{\Gamma}\left(E_{\mathcal{F}} \Gamma, \mathbb{K}(\mathbb{Z})\right) \rightarrow K_{*}(\mathbb{Z}[\Gamma]) .
$$

Received 15 February 2007; published online 9 December 2007.

2000 Mathematics Subject Classification 57R67, 57R19, 19D50 (primary).

This work was partially supported by NSF grants DMS 0405884 and DMS 0604878. 
If $A \otimes \mathbb{Q}$ is injective in (1.3), we say that the Novikov conjecture holds for $\Gamma$; and if $A$ is injective, we say that the generalized integral Novikov conjecture in algebraic $K$-theory holds for $\Gamma$.

Among discrete subgroups of Lie groups and related subgroups of algebraic groups that contain torsion elements, the generalized integral Novikov conjectures in both $K$ - and $L$-theories have been shown to hold for the following classes of groups:

(1) $S$-arithmetic subgroups of reductive algebraic groups defined over a global field and of rank zero, where by a global field - it means either a number field (that is, a finite extension of $\mathbb{Q}$ ) or the function field of a projective curve over a finite field [32];

(2) arithmetic subgroups of reductive algebraic groups over number fields [31];

(3) discrete subgroups of virtually connected Lie groups [2].

A natural problem is to prove this generalized integral Novikov conjecture for $S$-arithmetic subgroups of all algebraic groups defined over all global fields without any rank restriction on $\mathbf{G}$ or $\mathbf{G}$ being reductive. The class of $S$-arithmetic subgroups is important in the rigidity theory, cohomology theory of discrete groups and number theory. See $[\mathbf{3}, \mathbf{4}, \mathbf{8}, \mathbf{1 1}, \mathbf{1 3}$, Chapter 7; 39, 41, 42, 48, 49] for various results on $S$-arithmetic groups and more references.

In this paper, in fact, we prove that the generalized integral Novikov conjecture holds for a wider class than the class of $S$-arithmetic groups.

Theorem 1.1. Let $k$ be a global field, that is, either a number field (that is, a finite extension of $\mathbb{Q}$ ), or the function field of a projective curve over a finite field $\mathbb{F}_{q}$ (that is, a finite separable extension of $\left.\mathbb{F}_{q}(t)\right)$. Let $\Gamma \subset \mathrm{GL}(n, k)$ be any finitely generated subgroup. Then the generalized integral Novikov conjecture in both $K$ - and $L$-theories holds for $\Gamma$.

A special case is the following result.

Corollary 1.2. If $\Gamma \subset \operatorname{GL}(n, \overline{\mathbb{Q}})$ is any finitely generated subgroup, where $\overline{\mathbb{Q}}$ is the algebraic closure of $\mathbb{Q}$, then the generalized integral Novikov conjecture in both $K$ - and $L$ theories holds for $\Gamma$.

Proof. Let $\gamma_{1}, \ldots, \gamma_{m}$ be a set of generators of $\Gamma$. Then, there exists a number field $k$ such that $\gamma_{1}, \ldots, \gamma_{m} \in \mathrm{GL}(n, k)$, and hence, $\Gamma \subset \mathrm{GL}(n, k)$. Then the corollary follows from Theorem 1.1.

THEOREM 1.3. If $\mathbf{G}$ is an algebraic group defined over a global field $k$, then the generalized integral Novikov conjecture holds for every $S$-arithmetic subgroup of $\mathbf{G}(k)$.

REMARK 1.4. If $\mathbf{G}$ is reductive and $k$ is a number field, or $k$ is a function field and the $k$-rank of $\mathbf{G}$ is zero, then every $S$-arithmetic subgroup is finitely generated. In other cases, it is not necessarily finitely generated. In fact, over function fields, the finiteness properties of $S$ arithmetic subgroups are weaker. For example, it was conjectured that $S$-arithmetic subgroups are not of type $F P_{\infty}$; instead, it is not even of type $F P_{r}$, where $r$ is the S-rank of $\mathbf{G}$ which is the total sum of the ranks of $\mathbf{G}$ over the completions of $k$ over the places in $S$. These conjectures are now proved in [15, Theorems 1.1 and 1.2]. The precise value of $r$ such that arithmetic subgroups are of type $F P_{r-1}$ but not of type $F P_{r}$ is not completely determined yet. (See [3, p. $28 ; 14]$ and also $[4,13$, Chapter $7, \S 2, \S 4$, p. $197 ; 49]$ for more details on these conjectures and related references.)

This conjecture on type $F P_{r}$ implies that for such an $S$-arithmetic subgroup $\Gamma$, it is not known if $H_{i}(\Gamma, \mathbb{Z})$ is finitely generated in every degree $i$. In particular, the finite generation conditions in the celebrated result on the Novikov conjecture in $K$-theory in [6] are not satisfied. 
Therefore, even the rational injectivity of the assembly map in $K$-theory, that is, in (1.3), is new for such a natural class of groups, which contains $\operatorname{SL}\left(n, \mathbb{F}_{p}[t]\right)$, where $\mathbb{F}_{p}$ is a finite field, $t$ is a free variable, and $\mathbb{F}_{p}[t]$ is contained in the function field $\mathbb{F}_{p}(t)$ of $\mathbb{P}^{1}$ over $\mathbb{F}_{p}$.

On the other hand, the rational injectivity in $L$-theory for these $S$-arithmetic subgroups over function fields follows from the result in [27].

REMARK 1.5. It is worthwhile to point out that if $\mathbf{G}$ is an algebraic group defined over a function field $k$ and of positive $k$-rank, then $\mathbf{G}(k)$ does not admit torsion-free $S$-arithmetic subgroups. Hence, no such $S$-arithmetic subgroup $\Gamma$ admits a finite $B \Gamma$, or equivalently a $\Gamma$ cofinite $E \Gamma$, the universal space for proper and free actions. By the above remark and results in $[10,12]$, such a $\Gamma$ does not even admit a $\Gamma$-cofinite $E_{\mathcal{F}} \Gamma$. This is the reason that the generalized integral Novikov conjecture for this natural class of groups could not be handled by the criterions (or methods) in $[1,17,20,21,46,47,51]$.

Both Theorems 1.1 and 1.3 are special cases of the following result.

TheOREM 1.6. Let $G_{i}, i=1, \ldots, m$ be a finite collection of groups, where each $G_{i}$ is either a linear real Lie group, that is, a Lie group contained in some $\operatorname{GL}(n, \mathbb{R})$, or an algebraic group $\mathbf{G}\left(k_{\nu}\right) \subset \mathrm{GL}\left(n, k_{\nu}\right)$, where $k$ is a global field and $\nu$ is a finite place of $k$, and $k_{\nu}$ is the completion of $k$ with respect to a norm associated with $\nu$. Let $\Gamma \subset \prod_{i=1}^{m} G_{i}$ be any discrete subgroup. Then the generalized integral Novikov conjecture in both $K$ - and $L$-theories holds for $\Gamma$.

REMARK 1.7. An interesting special case of the above theorem is when $m=1$. Then any discrete subgroup of a locally compact linear group as above, for example, either a linear real Lie group or a $p$-adic Lie group, satisfies the generalized integral Novikov conjecture. On the other hand, considering groups of different types at the same time is important. For example, $\mathrm{GL}\left(n, \mathbb{Z}\left[\frac{1}{p}\right]\right)$, where $p$ is a prime number, is not a discrete subgroup of either $\mathrm{GL}(n, \mathbb{R})$ or $\mathrm{GL}\left(n, \mathbb{Q}_{p}\right)$, but rather a discrete subgroup of the product $\mathrm{GL}(n, \mathbb{R}) \times \mathrm{GL}\left(n, \mathbb{Q}_{p}\right)$. This point of view of treating multiple places (or primes) of $\mathbb{Q}$ at the same time, or more generally a global field, is natural and important in number theory and the rigidity theory of lattices; see $[\mathbf{3 9}, \mathbf{4 1}]$.

Theorem 1.6 follows from Theorem 1.9. Recall that a geodesic (length) space is called a CAT(0)-space if every triangle in it is thinner than a corresponding triangle in $\mathbb{R}^{2}$ of the same side lengths. It is well known that a simply connected complete Riemannian manifold of nonpositive curvature is a proper CAT(0)-space. The following result is also well known.

Proposition 1.8. The Bruhat-Tits buildings of reductive algebraic groups over locally compact, totally disconnected fields are also proper CAT(0)-spaces.

In fact, the Bruhat-Tits buildings are proper metric spaces and Euclidean buildings, that is, buildings whose apartments are isometric to Euclidean spaces. It is known (see [13, pp. 153155; 9, pp. 342-346, Theorem 10A.4]) that an Euclidean building is a CAT(0)-space, and hence, these Bruhat-Tits buildings are proper CAT(0)-spaces. The basic idea of the proof is as follows. Let $\Delta$ be such a building. For any apartment $E$ and a chamber $C$ in $E$, there is a retraction $\rho_{E, C}$ of $\Delta$ onto $E$ such that it maps every apartment $E^{\prime}$ containing $C$ onto $E$ and acts as the identity map on $E^{\prime} \cap E \supseteq C$. The crucial point is to observe that $\rho_{E, C}$ is distance decreasing with respect to the Tits metric $d$, that is, $d\left(\rho_{E, C}(x), \rho_{E, C}(y)\right) \leqslant d(x, y)$ for all $x, y \in \Delta$, and $d\left(\rho_{E, C}(x), \rho_{E, C}(y)\right)=d(x, y)$ if $x$ or $y$ belongs to $C$. (See also $[\mathbf{9}, \mathbf{3 5}$, Chapter 2] for comprehensive discussions on $\operatorname{CAT}(0)$-spaces.) 
Theorem 1.9. Let $X$ be a proper $C A T(0)$-space, and a discrete group $\Gamma$ act isometrically and properly on it. Suppose $X$ has the structure of a $\Gamma-C W$-complex and its dimension is finite. If the asymptotic dimension of $X$ is finite, asd $X<+\infty$, then the generalized integral Novikov conjecture in both $K$ - and $L$-theories holds for $\Gamma$.

REMARK 1.10. It is perhaps worthwhile to point out that in Theorem 1.9, the existence of an isometric and proper action of $\Gamma$ on a proper $\mathrm{CAT}(0)$-space with even compact quotient does not necessarily imply that the integral Novikov conjecture holds for $\Gamma$. The existence of the structure of a $\Gamma$-CW-complex on $X$ is important. Given the difficulties in proving the existence of equivariant triangulations of $\Gamma$-manifolds, when $\Gamma$ acts with some fixed points, in [28] (see also some discussions in [32]), this latter condition on $X$ is nontrivial.

REMARK 1.11. In the Novikov conjectures in (1.2) and (1.3) and the above theorems, we could replace the ring $\mathbb{Z}$ of integers by more general rings $R$, that is, we have the assembly maps:

$$
A: H_{*}^{\Gamma}\left(E_{\mathcal{F}} \Gamma, \mathbb{L}^{\langle-\infty\rangle}(R)\right) \rightarrow L_{*}^{\langle-\infty\rangle}(R[\Gamma]), \quad A: H_{*}^{\Gamma}\left(E_{\mathcal{F}} \Gamma, \mathbb{K}(R)\right) \rightarrow K_{*}(R[\Gamma]) .
$$

For the integral Novikov conjecture in L-theory, we need to assume further that $R$ is a ring with involution. For the validity of the generalized integral Novikov conjecture in L-theory, [2, Theorem B] (see Theorem 2.2 below) requires that for every finite subgroup $G$ of $\Gamma$, the algebraic groups $K_{-i}(R[G])=0$ when $i \gg 1$. By [19], this is satisfied when $R=\mathbb{Z}$. Therefore, for simplicity, we only deal with (or rather state the results only for) the ring $\mathbb{Z}$ in this paper.

\section{Proofs of Theorems 1.9, 1.6, 1.3 and 1.1}

In this section, we recall that some definitions and the general results of [2, Theorems A and $\mathrm{B}$ ], and use them to prove the theorems stated in the previous section.

More specifically, we first introduce the notion of uniform $\mathcal{F}$-contractiblity of a metric space. Combined with the notion of the asymptotic dimension of a metric space, we state $[\mathbf{2}$, Theorems $\mathrm{A}$ and B]. To use this result to prove Theorem 1.9 and, hence, Theorem 1.6, the crucial points are as follows.

(1) Observe that symmetric spaces and Bruhat-Tits buildings, and hence their products, are proper $\mathrm{CAT}(0)$-spaces.

(2) Apply the Cartan fixed point theorem (or rather a generalization) to proper $\operatorname{CAT}(0)$ spaces to prove the desired uniform $\mathcal{F}$-contractibility.

Then we recall that the notion of $S$-arithmetic subgroups and apply Theorem 1.6 to prove Theorem 1.3 and then Theorem 1.1.

Let $\Gamma$ be a discrete group, and $\mathcal{F}$ the family of all finite subgroups of $\Gamma$.

Definition 2.1. Let $(X, d)$ be a metric space, and $\Gamma$ act isometrically and properly on $X$. Then $X$ is called uniformly $\mathcal{F}$-contractible if for any $G \in \mathcal{F}$, that is, a finite subgroup of $\Gamma$, and every $r>0$, there exists $s>0$ such that for every $G$-invariant subset $\Omega$ of $X$ with diameter less than $r$, the inclusion $\Omega \hookrightarrow B(\Omega, s)$ is $G$-equivariantly null homotopic, where $B(\Omega, s)=\{x \in X \mid d(\Omega, x) \leqslant s\}$ is the $s$-neighborhood of $\Omega$.

Recall that the asymptotic dimension of a metric space $(X, d)$, denoted by asd $X$, was first introduced by Gromov [26] and is defined to be the least integer $n$ such that for every $r>0$ (meant to be large), there exists a covering of $X$ by uniformly bounded sets $\left\{U_{i}\right\}$ such that for every $x \in X$, the metric ball $B(x, r)$ meets at most $n+1$ sets from the covering $\left\{U_{i}\right\}$.

One of the main results in [2, Theorems A and B] is the following. 
Theorem 2.2. Let $\Gamma$ be a discrete group. Assume that there exists a finite dimensional $\Gamma$-CW-complex as the universal space $E_{\mathcal{F}} \Gamma$ (also often denoted by $\underline{E} \Gamma$ ) for proper $\Gamma$-actions, and assume that $E_{\mathcal{F}} \Gamma$ admits a $\Gamma$-invariant metric with respect to which $E_{\mathcal{F}} \Gamma$ is uniformly $\mathcal{F}$-contractible and has finite asymptotic dimension, asd $E_{\mathcal{F}} \Gamma<+\infty$. Then the generalized integral Novikov conjecture in both $K$ - and $L$-theories holds for $\Gamma$.

Remark 2.3. As pointed out in Remark 1.11 above, $[\mathbf{2}$, Theorems A and B] works for more general rings $R$. In this case, for the L-theory, it is required that for every finite subgroup $G \subset \Gamma, K_{-i}(R[G])=0$ for $i \gg 1$. By [19], this is satisfied when $R=\mathbb{Z}$.

Remark 2.4. The above theorem in $[\mathbf{2}]$ generalizes the results in $[\mathbf{1}, \mathbf{1 6}, \mathbf{2 0}, \mathbf{2 1}, \mathbf{4 6}, \mathbf{4 7}$, 51]. Unlike these papers, $\Gamma$ is not required to be finitely generated in the above theorem. The important point is that $E_{\mathcal{F}} \Gamma$ has finite dimension, but no compactness of the quotient $\Gamma \backslash E_{\mathcal{F}} \Gamma$ is required.

On the other hand, if $\Gamma \backslash E_{\mathcal{F}} \Gamma$ is compact and the $\Gamma$-action is metrically proper, then $\Gamma$ is finitely generated, and hence, asd $\Gamma$ is well defined and is equal to asd $E_{\mathcal{F}} \Gamma$. In fact, let $\Omega \subset E_{\mathcal{F}} \Gamma$ be a compact subset such that $\Gamma \Omega=E_{\mathcal{F}} \Gamma$. Let $\gamma_{1}, \ldots, \gamma_{m}$ be all the elements $\gamma$ of $\Gamma$ such that $\gamma \Omega \cap \Omega \neq \emptyset$. By the properness of the action, there are only finitely many of them. Then, it can be shown easily that $\gamma_{1}, \ldots, \gamma_{m}$ generate $\Gamma$.

Remark 2.5. In Theorem 2.2, the usual requirement in $[\mathbf{1}, \mathbf{1 6}, \mathbf{2 0}, \mathbf{2 1}, \mathbf{4 6}, \mathbf{4 7}, \mathbf{5 1}]$ that there is a $\Gamma$-cofinite $\mathrm{CW}$-complex model for $E_{\mathcal{F}} \Gamma$ is replaced by the existence of a finite dimensional $E_{\mathcal{F}} \Gamma$. This is important for many classes of groups. As pointed out in Remark 1.5, this is essential for the application to $S$-arithmetic subgroups of algebraic groups of positive rank over function fields such as $S L\left(n, \mathbb{F}_{p}[t]\right)$, which form a natural class of groups and have been intensively studied by various people (see $[\mathbf{3}, \mathbf{4}, \mathbf{1 3}$, Chapter VII]). Another source of examples of $\Gamma$ with a finite dimensional $E_{\mathcal{F}} \Gamma$ but no $\Gamma$-cofinite $\mathrm{CW}$-complex $E_{\mathcal{F}} \Gamma$ arises as follows. If $\Gamma$ has a $\Gamma$-cofinite $\mathrm{CW}$-complex model for $E_{\mathcal{F}} \Gamma$, then for an arbitrary subgroup $\Gamma^{\prime}$ of $\Gamma$, it is expected that $\Gamma^{\prime}$ often does not have a $\Gamma^{\prime}$-cofinite $\mathrm{CW}$-complex model. See [9, Chapter $\Gamma$, §5, Example 5.4] for such examples. In [31], every arithmetic subgroup $\Gamma$ of a reductive algebraic group defined over $\mathbb{Q}$ has a $\Gamma$-cofinite $C W$-complex model of $E_{\mathcal{F}} \Gamma$ given by the Borel-Serre (partial) compactification of symmetric spaces in [7]. On the other hand, a random subgroup of such an arithmetic subgroup $\Gamma$ will probably not inherit such a finiteness property.

Proof of Theorem 1.9. It suffices to show that $X$ is uniformly $\mathcal{F}$-contractible. Let $G$ be a finite subgroup of $\Gamma$, and $\Omega$ a $G$-invariant subset with diameter less than $r$. We claim that we can take $s=2 r$ and the inclusion $\Omega \hookrightarrow B(\Omega, 2 r)$ is $G$-equivariantly null homotopic.

Let $\bar{\Omega}$ be the closure of $\Omega$. Clearly, it is also $G$-invariant. Let $C H(\bar{\Omega})$ be the convex hull of $\bar{\Omega}$. Then it is also $G$-invariant. Clearly, $C H(\bar{\Omega}) \subset B(\bar{\Omega}, r)=B(\Omega, r)$.

Since $X$ is a proper CAT(0)-space by [35, Corollary 2.3.1, p. 58], it is a uniform, simply connected Busemann nonpositively curved space in the sense of [35, Definition 2.2.4, p. 44; Definition 2.2.6, p. 50]. Hence, by the generalization in [35, Corollary 3.2.1, p. 66] of the famous Cartan fixed point theorem (see [22, Theorem 1.4.6] for example), there is a fixed point of $G$ in $C H(\bar{\Omega})$. Briefly, for any point $x \in C H(\bar{\Omega})$, the center of mass of the $G$-orbit $G x$ is fixed by $G$. In [35, Lemma 3.3.4, p. 68], this center of mass is contained in their convex hull, and hence, it is contained in $C H(\bar{\Omega})$.

Since $X$ is a CAT(0)-space, every point in $X$ can be connected to $x_{0}$ by a unique geodesic ray, and $X$ can be contracted to the point $x_{0}$ along these geodesics. This implies that $C H(\bar{\Omega})$ and $\Omega$ can be contracted in $B(\Omega, 2 r)$ to $x_{0}$ along these rays. Since $G$ fixes $x_{0}$ and acts on $X$ by isometry, this contraction of $\Omega$ to $x_{0}$ is $G$-equivariant. 
This shows that $X$ is uniformly $\mathcal{F}$-contractible and Theorem 2.2 can be applied to prove Theorem 1.9.

To prove other theorems, we need a model for $E_{\mathcal{F}} \Gamma$ satisfying the conditions in Theorem 1.9.

Proof of Theorem 1.6. We construct a model of $E_{\mathcal{F}} \Gamma$ satisfying the conditions in Theorem 1.9 by using either the symmetric space of $\mathrm{GL}(n, \mathbb{R})$ or the Bruhat-Tits building of $\operatorname{GL}\left(n, k_{\nu}\right)$. In fact, for each $i=1, \ldots, m$, if $G_{i} \subset \mathrm{GL}(n, \mathbb{R})$, let $X_{i}$ be the symmetric space $\mathrm{GL}(n, \mathbb{R}) / O(n)$, which is simply connected and nonpositively curved; if $G_{i} \subset \mathrm{GL}\left(n, k_{\nu}\right)$, where $\nu$ is a finite place of $k$ (recall that a place of $k$ is an equivalence class of valuations of $k$ ) and $k_{\nu}$ is a locally compact, totally disconnected field with a finite residue field, let $X_{i}$ be the Bruhat-Tits building associated with the group $\mathrm{GL}\left(n, k_{\nu}\right)$. It is known that the Bruhat-Tits building of $\operatorname{GL}\left(n, k_{\nu}\right)$ has a canonical metric, the so-called Tits metric whose restriction to each apartment is isometric to an Euclidean space $\mathbb{R}^{r}$. By construction, $\operatorname{GL}\left(n, k_{\nu}\right)$ acts isometrically and properly on the building $X_{i}$ (see $[\mathbf{1 3}, \mathbf{3 4}]$ for basic facts about buildings and other references).

Define

$$
X=\prod_{i=1}^{m} X_{i}
$$

and endow it with the product metric of the distance function from the invariant Riemannian metric of the symmetric spaces and the Tits metrics of the buildings. Since $\Gamma$ is a discrete subgroup of $\prod_{i=1}^{m} G_{i}$, and each $G_{i}$ acts isometrically and properly on $X_{i}$, it follows that $\Gamma$ acts isometrically and properly on $X$.

It is well known that if $X_{i}$ is a simply connected complete Riemannian manifold of nonpositive curvature, then it is a proper CAT(0)-space; and if $X_{i}$ is a Bruhat-Tits building, it is also a proper CAT(0)-space (see Proposition 1.8 and [9, pp. 342-346, Theorem 10A.4; 13, pp. 153155]). A basic simple observation is that the product $X=\prod_{i=1}^{m} X_{i}$ is also a proper $\operatorname{CAT}(0)$ space (see [9, p. 167]). In particular, it is contractible by contracting along rays from a fixed basepoint.

For any finite subgroup $G \subset \Gamma$, the fixed point set $X^{G}$ is a totally geodesic subspace and is, hence, contractible. This implies that $X$ is a $E_{\mathcal{F}} \Gamma$-space (see [37]).

Next we show that $X$ has the structure of a $\Gamma$-CW-complex. If $X_{i}$ is a Bruhat-Tits building, by construction, it is a simplicial complex on which $G_{i}$ acts simplicially, and hence, it has a canonical CW-complex structure invariant under $G_{i}$. Therefore, if there is no factor $X_{i}$ given by the symmetric space of $\mathrm{GL}(n, \mathbb{R})$, then $X$ has the structure of a $\Gamma$-CW-complex. If $X$ does not contain any factor given by a Bruhat-Tits building, then the existence of a $\Gamma$-CW-complex structure on $X$ follows from the existence of equivariant triangulations in [28, Theorems I and II].

On the other hand, assume that $X$ contains both the symmetric space factors and the Bruhat-Tits building factors. Then we need to use arguments similar to those in [32, Proposition 2.13]. For simplicity, assume that $m=2$ and $X=X_{1} \times X_{2}$, where $X_{1}$ is the symmetric space of $\operatorname{GL}(n, \mathbb{R})$, and $X_{2}$ is the Bruhat-Tits building of $G L\left(n, k_{\nu}\right)$, where $k_{\nu}$ is a local field.

By assumption, $\Gamma$ acts properly on $X$. This implies that for any simplex $\sigma$ of the building $X_{2}$, the stabilizer $\Gamma_{\sigma}=\{\gamma \in \Gamma \mid \gamma \sigma=\sigma\}$ acts properly on $X_{1} \times \sigma$. By using the equivariant triangulation of $X_{1} \times \sigma$ with respect to $\Gamma_{\sigma}$ and modify suitably the CW-complex structures on the boundary $X_{1} \times \partial \sigma$, where $\partial \sigma$ is the boundary of $\sigma$, we can show as in [32, Proposition $2.13]$ that $X$ has the structure of a $\Gamma$-CW-complex.

Clearly, $\operatorname{dim} X<+\infty$. To finish the proof, we need to show asd $X<+\infty$. This follows from the following result. 
Proposition 2.6. If $X_{i}$ is either a symmetric space or a Bruhat-Tits building as above, then asd $X_{i}<+\infty$.

Proof. If $X_{i}$ is a symmetric space, then the proof follows from [18, Theorem $3.5 ; \mathbf{5}$ ].

If $X_{i}$ is a Bruhat-Tits building, then we can use [40, Theorem 3.21] to show that asd $X_{i}<$ $+\infty$. In fact, $G L(n) \subset S L(n+1)$ over $k$. Hence, $G L\left(k_{\nu}\right) \subset S L\left(n+1, k_{\nu}\right)$. Let $\Delta_{\nu}$ be the Bruhat-Tits building of $S L\left(n+1, k_{\nu}\right)$. In [36, Theorem 2.2.1], there is an isometric embedding of $X_{i}$ into $\Delta_{\nu}$. Hence, $X_{i}$ can be identified isometrically with a subspace of $\Delta_{\nu}$. Therefore, it suffices to prove asd $\Delta_{\nu}<+\infty$, which is precisely the result in [40, Theorem 3.21].

Briefly, the proof of the bound asd $\Delta_{\nu}<+\infty$ in [40, Theorem 3.21] goes as follows. Define a length function $\ell$ on $S L\left(n+1, k_{\nu}\right)$ by

$$
\ell(g)=-\min \left\{\nu_{\nu}\left(g_{i j}\right), \nu_{\nu}\left(g^{i j}\right)\right\}, \quad \text { where } g=\left(g_{i j}\right), g^{-1}=\left(g^{i j}\right) .
$$

This length function defines a pseudo-distance function $d$ on $S L\left(n+1, k_{\nu}\right)$ by $d(g, h)=\ell\left(g^{-1} h\right)$. Let $\mathcal{O}_{k_{\nu}}$ be the ring of integers of $k_{\nu}$. Then $\ell(g)=0$ if and only if $g \in S L\left(n+1, \mathcal{O}_{k_{\nu}}\right)$. This implies that the pseudo-distance $d$ on $S L\left(n+1, k_{\nu}\right)$ defines a distance on the quotient $S L\left(n+1, k_{\nu}\right) / S L\left(n+1, \mathcal{O}_{k_{\nu}}\right)$. Since $S L\left(n+1, k_{\nu}\right)$ acts isometrically on $\Delta_{\nu}$ and transitively on the set of chambers of $\Delta_{\nu}$ with the stabilizers of chambers equal to conjugates of a subgroup $I$ of $S L\left(n+1, \mathcal{O}_{k_{\nu}}\right)$ with finite index, it follows that $S L\left(n+1, k_{\nu}\right) / S L\left(n+1, \mathcal{O}_{k_{\nu}}\right)$ with the distance $d$ is coarsely equivalent to $\Delta_{\nu}$. Hence, it suffices to show that the asymptotic dimension of $S L\left(n+1, k_{\nu}\right) / S L\left(n+1, \mathcal{O}_{k_{\nu}}\right)$ with respect to $d$, or equivalently, the asymptotic dimension of $S L\left(n+1, k_{\nu}\right)$ with respect to the pseudo-distance $d$ is finite.

Let $B$ be the subgroup of upper triangular matrices of $S L\left(n+1, k_{\nu}\right)$. Then $S L\left(n+1, k_{\nu}\right)$ admits the following decomposition

$$
S L\left(n+1, k_{\nu}\right)=B S L\left(n+1, \mathcal{O}_{k_{\nu}}\right) .
$$

The restriction of $d$ to $B$ defines a pseudo-distance $d$ on $B$, and it suffices to show that the asymptotic dimension of $B$ is finite. Let $N$ be the subgroup of $B$ consisting of matrices with 1s on the diagonal, and $A$ the subgroup of diagonal matrices. Then $B=N A$.

The crucial observation is that the restriction of $d$ on $N$ is coarsely equivalent to an ultra metric, and hence, the asymptotic dimension of $N$ is equal to 0 [40, Lemma 3.20, Theorem 3.16]. Since $A$ is coarsely equivalent to an apartment in $\Delta_{\nu}$, which is isometric to $\mathbb{R}^{n}$, asd $A=n<$ $+\infty$. Then a result of Hurewicz type on asymptotic dimensions in [5] implies that asd $\Delta_{\nu}<\infty$.

Remark 2.7. In Proposition 2.6, it can be proved that asd $X_{i}=\operatorname{dim} X_{i}$ and, hence, asd $X=\operatorname{dim} X=n$. More generally, it can be shown that the asymptotic dimension of the Bruhat-Tits building of every reductive algebraic group defined over a locally compact field with a discrete valuation is equal to its dimension. Moreover, sharp bounds on the asymptotic dimension of $S$-arithmetic subgroups of reductive groups defined over a global field can also be obtained as for arithmetic subgroups in [29]. The details are contained in [33].

To prove Theorems 1.1 and 1.3, we need to embed $\Gamma$ into the product of groups of the type in Theorem 1.6.

Before proving Theorem 1.3, we first recall the notion of $S$-arithmetic subgroups. Let $k$ be a global field. If $k$ is a number field, let $S_{\infty}$ be the set of all infinite (archimedean) places of $k$. For example, when $k=\mathbb{Q}$, there is only one infinite place. If $k$ is a function field, there is no infinite place; in this case, we set $S_{\infty}$ to be an empty set. Let $S$ be a finite set of places of $k$ containing $S_{\infty}$. If $k=\mathbb{Q}$, we can take $S$ to correspond to $\left\{\infty, p_{1}, \ldots, p_{m}\right\}$, where each $p_{i}$ is a prime number. Let $S_{f}=S-S_{\infty}$. If $k$ is a function field, then $S_{f}=S$. 
For each place $\nu$ of $k$, denote an associated valuation of $k$ also by $\nu$ for convenience. Then the ring $\mathcal{O}_{k, S}$ of $S$-integers of $k$ is defined to be

$$
\mathcal{O}_{k, S}=\{x \in k \mid \nu(x) \geqslant 0, \text { for all } \nu \notin S\} .
$$

For example, if $k=\mathbb{Q}$ and $S=\left\{\infty, p_{1}, \ldots, p_{m}\right\}$, then $\mathcal{O}_{k, S}=\mathbb{Z}\left[\frac{1}{p_{1}}, \ldots, \frac{1}{p_{m}}\right]$, the ring of rational numbers $\frac{q}{p}, p, q \in \mathbb{Z}$, with $p$ only divisible by the primes $p_{1}, \ldots, p_{m}$; that is, the ring $\mathbb{Z}\left[\frac{1}{p_{1}}, \ldots, \frac{1}{p_{m}}\right]$ is obtained from $\mathbb{Z}$ by inverting these finitely many primes $p_{1}, \ldots, p_{m}$.

For each place $\nu$, let $k_{\nu}$ be the completion of $k$ with respect to a norm associated with $\nu$. When $\nu$ is an infinite place, $k_{\nu}=\mathbb{R}$ or $\mathbb{C}$. If $\nu \in S_{f}$, that is, a finite place, then $k_{\nu}$ is a locally compact, totally disconnected field with a finite residue. For example, if $k=\mathbb{Q}$ and $\nu$ corresponds to a prime number $p$, then $k_{\nu}=\mathbb{Q}_{p}$, the field of $p$-adic numbers, and the residue field is $\mathbb{Z} / p \mathbb{Z}=\mathbb{F}_{p}$, the finite field with $p$ elements.

Let $\mathbf{G} \subset \mathrm{GL}(n)$ be an algebraic group defined over $k$. Then a subgroup $\Gamma$ of $\mathbf{G}(k)$ is called an $S$-arithmetic subgroup if it is commensurable with $\mathbf{G}\left(\mathcal{O}_{k, S}\right)=\mathbf{G}(k) \cap \mathrm{GL}\left(n, \mathcal{O}_{k, S}\right)$, that is, the intersection $\Gamma \cap \mathbf{G}\left(\mathcal{O}_{k, S}\right)$ is of finite index in both $\Gamma$ and $\mathbf{G}\left(\mathcal{O}_{k, S}\right)$.

An important example of $S$-arithmetic subgroups over number fields is $\operatorname{SL}\left(n, \mathbb{Z}\left[\frac{1}{p_{1}}, \ldots, \frac{1}{p_{m}}\right]\right)$, which is clearly not a discrete subgroup of the real Lie group $\operatorname{GL}(n, \mathbb{R})$; and an important example over function fields is $\operatorname{SL}\left(n, \mathbb{F}_{p}[t]\right)$, where $t$ is a free variable and $\mathbb{F}_{p}$ is a finite field, and the function field is $\mathbb{F}_{p}(t)$.

Proof of Theorem 1.3. Define

$$
G_{S}=\prod_{\nu \in S} \mathbf{G}\left(k_{\nu}\right)
$$

We note that only when $S_{f}=\emptyset, G_{S}$ is a real Lie group. It is known that an $S$-arithmetic subgroup $\Gamma$ can be embedded diagonally into $G_{S}$ as a discrete subgroup. Clearly, $G_{S}$ is a product group of the type in Theorem 1.6, and hence, by Theorem 1.6 the generalized integral Novikov conjecture holds for $\Gamma$.

Proof of Theorem 1.1. Let $\Gamma$ be a finitely generated subgroup of $\operatorname{GL}(n, k)$, where $k$ is a global field. Let $\left\{\gamma_{1}, \ldots, \gamma_{m}\right\}$ be a symmetric set of generators of $\Gamma$, that is, it also contains the inverses $\gamma_{1}^{-1}, \ldots, \gamma_{m}^{-1}$. Then there exists a finite set $S$ of places of $k$ such that the matrix entries of every generator $\gamma_{i}$ under the embedding into $\operatorname{GL}(n, k)$ are S-integers. (When $k=\mathbb{Q}$, we include all those primes that appear in the denominators of the entries of $\gamma_{i}$ into $S$.) Then $\Gamma$ is contained in the $S$-arithmetic subgroup $\operatorname{GL}\left(n, \mathcal{O}_{k, S}\right)$. Therefore, Theorem 1.6 can be applied and the generalized integral Novikov conjecture holds for $\Gamma$. Theorem 1.1 is proved.

Finally, we give a proof of the statement mentioned in the introduction that the integral Novikov conjecture in the surgery theory implies the stable Borel conjecture. The proof of Proposition 2.8 is provided by Shmuel Weinberger and is related to [23, Corollary B and its proof on p. 207].

Proposition 2.8. If the assembly map $A^{\langle-\infty\rangle}$ in (1.1) is injective for $\Gamma$, then the stable Borel conjecture holds for $\Gamma$, that is, if two closed aspherical manifolds $M^{n}$ and $N^{n}, n \geqslant 5$ have the fundamental group equal to $\Gamma$, then $M \times \mathbb{R}^{3}$ and $N \times \mathbb{R}^{3}$ are homeomorphic.

Proof. The surgery exact sequence for topological manifolds is an exact sequence of abelian groups:

$$
\cdots \rightarrow L_{n+1}(\mathbb{Z} \Gamma) \rightarrow \mathcal{S}^{T O P}(M) \rightarrow[M, G / T O P] \stackrel{A\langle 1\rangle}{\longrightarrow} L_{n}(\mathbb{Z} \Gamma)
$$


such that

$$
\operatorname{im}\left(L_{n+1}(\mathbb{Z} \Gamma) \rightarrow \mathcal{S}^{T O P}(M)\right)=\operatorname{ker}\left(\mathcal{S}^{T O P}(M) \rightarrow[M, G / T O P]\right) \subseteq \mathcal{S}^{T O P}(M)
$$

is the subgroup of the homotopy equivalences $N \rightarrow M$ for which there exists a normal cobordism to the identity $M \rightarrow M$. The map

$$
A\langle 1\rangle:[M, G / T O P]=H_{n}(M, \mathbb{L} .\langle 1\rangle(\mathbb{Z})) \rightarrow L_{n}(\mathbb{Z} \Gamma)
$$

is the assembly map on the 1 -connective quadratic L-spectrum $\mathbb{L} .\langle 1\rangle(\mathbb{Z})$ with 0 th space homotopy equivalent to $G / T O P$. This assembly can be factorized as

$$
A\langle 1\rangle:[M, G / T O P] \rightarrow[M, \mathbb{Z} \times G / T O P]=H_{n}(M, \mathbb{L}(\mathbb{Z})) \stackrel{A}{\longrightarrow} L_{n}(\mathbb{Z} \Gamma)
$$

with $\mathbb{L}(\mathbb{Z})$ the 4-periodic quadratic L-spectrum of $\mathbb{Z}$ [43, Theorem 18.5 and Chapter 25], and the map $[M, G / T O P] \rightarrow[M, \mathbb{Z} \times G / T O P]$ is injective. (Note that $A$ is yet another assembly map.)

Since the negative K-groups of $\mathbb{Z}$ all vanish, $\mathbb{L}(\mathbb{Z})=\mathbb{L}^{\langle-\infty\rangle}(\mathbb{Z})$, and hence

$$
[M, \mathbb{Z} \times G T O P]=H_{n}(M, \mathbb{L}(\mathbb{Z}))=H_{n}(B \Gamma, \mathbb{L}(\mathbb{Z}))=H_{n}\left(B \Gamma, \mathbb{L}^{\langle-\infty\rangle}(\mathbb{Z})\right) .
$$

(Note that $M$ is aspherical with $\pi_{1}(M)=\Gamma$ and can be taken as a $B \Gamma$-space.) There is a map $L_{n}(\mathbb{Z} \Gamma) \rightarrow L_{n}^{\langle-\infty\rangle}(\mathbb{Z} \Gamma)$ (see [44]) such that the assembly map $A^{\langle-\infty\rangle}$ factors as

$$
A^{\langle-\infty\rangle}: H_{n}\left(B \Gamma, \mathbb{L}^{\langle-\infty\rangle}(\mathbb{Z})\right)=H_{n}(M ; \mathbb{L}(\mathbb{Z})) \stackrel{A}{\longrightarrow} L_{n}(\mathbb{Z} \Gamma) \rightarrow L_{n}^{\langle-\infty\rangle}(\mathbb{Z} \Gamma) .
$$

Thus, if $A^{\langle-\infty\rangle}$ is injective, then both $A$ and $A\langle 1\rangle$ are also injective. Since every homotopy equivalence $N \rightarrow M$ is mapped to zero in $L_{n}(\mathbb{Z} \Gamma)$, the injectivity of $A\langle 1\rangle$ implies that it belongs to $\operatorname{ker}\left(\mathcal{S}^{T O P}(M) \rightarrow[M, G / T O P]\right)$ and is normal cobordant to the identity $M \rightarrow M$ as pointed out above in $(2.1)$.

By the assumption in the proposition, the manifolds $M$ and $N$ are aspherical with the same fundamental group $\Gamma$, and consequently, there is a homotopy equivalence $N \rightarrow M$. Therefore, $N$ and $M$ are cobordant by a normal cobordism $W$.

Let $D$ be a closed three-dimensional ball. Then $W \times D$ is a normal corbordism between $M \times D$ and $N \times D$. Note that the boundary of $W \times D$ is the union of $M \times D, N \times D$, and $W \times S$, where $S$ is the boundary of $D$, a two-dimenmsional sphere. Since the fundamental groups of $W \times S$ and $W \times D$ are the same, Wall's $\pi-\pi$ theorem allows us to do surgery to change $W \times D$ into a h-cobordism $H$ between $M \times D$ and $N \times D$. Removing a part of the boundary of $H$ gives a proper h-cobordism $K$ between $M \times \mathbb{R}^{3}$ and $N \times \mathbb{R}^{3}$. Since $M$ is compact and connected, and $\mathbb{R}^{3}$ is simply connected at infinity, the relevant Whitehead group for proper h-corbordisms on $M \times \mathbb{R}^{3}$ vanishes, and the proper h-corbordism theorem in [50] (see also [25, pp. 511-512]) implies that $K$ is a cylinder, and hence, $M \times \mathbb{R}^{3}$ is homeomorphic to $N \times \mathbb{R}^{3}$.

Remark 2.9. In Proposition 2.8, we need to impose the condition that $\operatorname{dim} M \geqslant 5$. This condition does not really affect the validity of the stable Borel conjecture for torsion-free groups $\Gamma$ in Theorems 1.3, 1.5, 1.8, 1.11 and Corollary 1.4. In fact, if $\Gamma$ satisfies the conditions in these results and, hence, the integral Novikov conjecture holds for it, then for $m \geqslant 3$, the integral Novikov conjecture is also valid for $\Gamma \times \mathbb{Z}^{m}$. Then we can argue as in [34, Corollary B and its proof on p. 207] that $M \times \mathbb{R}^{m}$ and $N \times \mathbb{R}^{m}$ are homeomorphic for all closed aspherical manifolds with $\pi_{1}$ equal to $\Gamma$.

Acknowledgements. I would like to thank David Rosenthal for sending me the paper [2], and Hyman Bass, Arthur Bartels and Wolfgang Lück for pointing out the reference [19], and Tom Farrell, Shmuel Weinberger and Andrew Ranicki for explaining to me patiently the relations between the integral Novikov conjecture and the stable Borel conjecture in Proposition 2.8, 
and providing the proof above. I would also like to thank Ralf Spatzier for a conversation on the Cartan fixed point theorem for CAT(0)-spaces, G. Prasad for carefully reading this paper and for several helpful comments and suggestions, and Pierre de la Harpe for pointing out the example in $[\mathbf{9}$, p. 483] in 2005, which is mentioned in Remark 2.5. Finally, I would like to thank the anonymous referee for carefully reading earlier versions of this paper and making several constructive suggestions.

\section{References}

1. A. BARtels, 'Squeezing and higher algebraic $K$-theory', $K$-Theory 28 (2003) 19-37.

2. A. Bartels and D. Rosenthal, 'On the $K$-theory of groups with finite asymptotic dimension', Preprint, 2006 arXiv:math.KT/0605088.

3. H. BeHR, 'Higher finiteness properties of $S$-arithmetic groups in the function field case I', Groups: Topological, combinatorial and arithmetic aspects, London Mathematical Society Lecture Note Series, 311 (Cambridge University Press, Cambridge, 2004).

4. H. BeHR, 'Arithmetic groups over function fields. I. A complete characterization of finitely generated and finitely presented arithmetic subgroups of reductive algebraic groups', J. Reine Angew. Math. 495 (1998) $79-118$.

5. G. Bell and A. Dranishnikov, 'A Hurewicz-type theorem for asymptotic dimension and applications to geometric group theory', Trans. Amer. Math. Soc. 358 (2006) 4749-4764.

6. M. Bökstedt, W. Hsiang and I. Madsen, 'The cyclotomic trace and algebraic $K$-theory of spaces', Invent Math. 111 (1993) 465-539.

7. A. Borel and J. P. Serre, 'Corners and arithmetic groups', Comment. Math. Helv. 48 (1973) 436-491.

8. A. Borel and J. P. Serre, 'Cohomologie dímmeubles et de groupes S-arithmétiques', Topology 15 (1976) $211-232$

9. M. Bridson and A. HAefliger, Metric spaces of non-positive curvature, Grundlehren der Mathematischen Wissenschaften 319 (Springer, Berlin, 1999).

10. K. Brown, 'Groups of virtually finite dimension', Homological group theory, London Mathematical Society Lecture Note Series 36 (Cambridge University Press, Cambridge, 1979) 27-70.

11. K. Brown, Cohomology of groups (Springer, Berlin, 1982).

12. K. Brown, 'Finiteness properties of groups', J. Pure Appl. Algebra 44 (1987) 45-75.

13. K. Brown, Buildings (Springer, Berlin, 1988).

14. K. Bux and K. Wortman, 'A geometric proof that $S L_{2}\left(Z\left[t, t^{-1}\right]\right)$ is not finitely presented', Algebraic Geom. Topol. 6 (2006) 839-852.

15. K. Bux and K. Wortman, 'Finiteness properties of arithmetic groups over function fields', Invent. Math. 167 (2007) 355-378.

16. G. Carlsson and E. Pedersen, 'Controlled algebra and the Novikov conjectures for $K$ - and $L$-theory', Topology 34 (1995) 731-758.

17. G. Carlsson and B. Goldfarb, 'The integral $K$-theoretic Novikov conjecture for groups with finite asymptotic dimension', Invent. Math. 157 (2004) 405-418.

18. G. CARlsson and B. Goldfarb, 'On homological coherence of discrete groups', J. Algebra 276 (2004) 502-514.

19. D. CARTer, 'Localization in lower algebraic K-theory', Commun. Algebra 8 (1980) 603-622.

20. S. Chang, S. Ferry and G. YU, 'Bounded rigidity of manifolds and asymptotic dimension growth', K-theory (2007) in press.

21. A. Dranishnikov, S. Ferry and S. Weinberger, 'An etale approach to the Novikov conjecture', Preprint 2005, math.GT/0509644.

22. P. Eberlein, Geometry of nonpositively curved manifolds, Chicago Lectures in Mathematics (University of Chicago Press, Chicago, 1996).

23. F. FARrell and W. C. Hsiang, 'On Novikov's conjecture for nonpositively curved manifolds. I', Ann. Math. 113 (1981) 199-209.

24. F. FARrell and J. LAFOnt, 'EZ-structures and topological applications', Comment. Math. Helv. 80 (2005) 103-121.

25. F. FArrell and J. Wagoner, 'Algebraic torsion for infinite simple homotopy types', Comment. Math. Helv. 47 (1972) 502-513.

26. M. Gromov, 'Asymptotic invariants of infinite groups', Geometric group theory (ed. A. Niblo and M. Roller; Cambridge University Press, Cambridge).

27. E. Guentner, N. Higson and S. Weinberger, 'The Novikov conjecture for linear groups', Math. Publ. d'IHES 101 (2005) 243-268.

28. S. Illman, 'Existence and uniqueness of equivariant triangulations of smooth proper $G$-manifolds with some applications to equivariant Whitehead torsion', J. Reine Angew. Math. 524 (2000) 129-183.

29. L. JI, 'Asymptotic dimension and the integral K-theoretic Novikov conjecture for arithmetic groups', J. Differential Geom. 68 (2004) 535-544.

30. L. JI, 'Large scale geometry, compactifications and the integral Novikov conjectures for arithmetic groups', Proceedings of the International Congress of Chinese Mathematicians, 2004, in press. 
31. L. JI, 'Integral Novikov conjectures and arithmetic groups containing torsion elements', Comm. Anal. Geom. 15 (2007) 99-123.

32. L. JI, 'Integral Novikov conjectures for $S$-arithmetic groups I', K-theory 38 (2007) 35-47.

33. L. JI, 'Integral Novikov conjectures for $S$-arithmetic groups II', Unpublished preprint.

34. L. JI, 'Buildings and their applications in geometry and topology', Asian J. Math. 10 (2006) 11-80.

35. J. Jost, Nonpositive curvature: Geometric and analytic aspects (Birkhäuser, Klosterberg, 1997).

36. E. Landvogt, 'Some functorial properties of the Bruhat-Tits building', J. Reine Angew. Math. 518 (2000) 213-241.

37. W. LüCK, 'Survey on classifying spaces of familes of subgroups', Infinite groups: Geometric, combinatorial and dynamical aspects, Progress in Mathematics 248 (Birkhäuser, Klosterberg, 2005) 269-322.

38. W. LüCK and H. REICH, 'The Baum-Connes and the Farrell-Jones conjectures in $K$ - and $L$-theory', Handbook of $K$-theory, vol. 1, 2 (Springer, Berlin, 2005) 703-842.

39. G. Margulis, Discrete subgroups of semisimple Lie groups (Springer, Berlin, 1991) x+388.

40. D. MAtsnev, 'The Baum-Connes conjecture and group actions on affine buildings', Thesis, Pennsylvania State University, University Park, 2005.

41. V. Platonov and A. Rapinchuk, Algebraic groups and number theory, Pure and Applied Mathematics 139 (Academic Press, New York, 1994).

42. G. Prasad, 'Semi-simple groups and arithmetic subgroups', Proceedings of the International Congress of Mathematicians (Mathematical Society of Japan, Tokyo, 1991) 821-832.

43. A. RANicki, 'Algebraic L-theory and topological manifolds', Cambridge Tracts in Mathematics 102 (Cambridge University Press, Cambridge, 1992).

44. A. Ranicki, Lower $K$ - and L-theory, London Mathematical Society Lecture Note Series 178 (Cambridge University Press, Cambridge, 1992).

45. J. Rosenberg, 'Review of The Novikov conjecture: Geometry and algebra by M. Kreck and W. Lück', Bull. Amer. Math. Soc. 43 (2006) 599-604.

46. D. Rosenthal, 'Splitting with continuous control in algebraic $K$-theory', $K$-Theory 32 (2004) 139-166.

47. D. Rosenthal, 'Continuous control and the algebraic L-theory assembly map', Forum Math. 18 (2006) 193-209.

48. J. P. SerRe, 'Arithmetic groups', Homological group theory (Cambridge University Press, Cambridge, 1979).

49. J. P. Serre, 'Cohomologie des groupes discrets', Prospects in Mathematics, Annals of Mathematics Studies 70 (Princeton University Press, Princeton, 1972) 77-169.

50. L. Siebenmann, 'Infinite simple homotopy types', Indag. Math. 32 (1970) 479-495.

51. G. YU, 'The Novikov conjecture for groups with finite asymptotic dimension', Ann. Math. 147 (1998) $325-355$.

\author{
Lizhen Ji \\ Department of Mathematics \\ University of Michigan \\ Ann Arbor, MI 48109 \\ $U S A$
}

